\title{
Shrinking the Reuse Distance: Spectrally-Efficient Radio Resource Management in D2D-Enabled Cellular Networks with Interference Alignment*
}

\author{
Hussain E. Elkotby \\ Khaled M. F. Elsayed \\ Mahmoud H. Ismail \\ Department of Electronics and Communications Engineering, Cairo University, Giza, Egypt 12613 \\ E-mails: \{khaled, mhismail\}@ieee.org \\ 'Department of Electronics and Communications Engineering, Port Said University, Port Said, Egypt 42523 \\ E-mail: hussain.elsaid@eng.psu.edu.eg
}

\begin{abstract}
Enabling underlay direct Device-to-Device (D2D) communication mode in future cellular networks has good potential for spectrally-efficient and low-latency support of local media services. Recently, it has become evident that shrinking the reuse distance over which wireless resources are reused is a key enabler for achieving high spectral efficiency. Moreover, Interference Alignment (IA) based transmission can enhance the capacity of a wireless network by providing more degrees of freedom. In this work, we exploit clustering of D2D users, frequency reuse over clusters and then using IA to enhance the sum rate. Specifically, we show that in a D2D environment, it is possible to achieve significant gains in attainable rates by constructing clusters of D2D pairs and reuse the available radio resources over the clusters. Moreover, within a cluster, it is possible to further enhance the spectral efficiency by constructing small-sized groups of D2D pairs over which IA is applied to offer additional degrees of freedom. We show that resource reuse over the clusters offer overall rate increase proportional to the number of formed clusters. In addition, interference alignment offers up to $33 \%$ increase in the overall rates in the high transmission power regimes compared to the normal Point-toPoint (P2P) communication.
\end{abstract}

Keywords-D2D; interference alignment; throughput enhancement, frequency reuse, clustering, grouping.

\section{INTRODUCTION}

Recently, direct device-to-device communication (D2D) as an underlay network to IMT-Advanced cellular networks [1] has been proposed. D2D represents a promising technique that is expected to provide efficient utilization of the available wireless spectrum. Moreover, this technique has been proposed as a new technology component for LTE-Advanced that is expected to provide access to the Internet and local services using licensed bands. In comparison, unlicensed spectrum operation of Bluetooth and WLAN causes uncertainty as to whether the spectrum and services are truly available.

D2D current research areas include the study of D2D and cellular users interference, which are discussed in [1] and [2], where a power control optimization and coordination mechanism is used. The concept behind this coordination mechanism is to select one of four different resource allocation modes; downlink resource sharing, uplink resource sharing, separate resource sharing and conventional cellular system mode. The results in [1] show that significant gains in the sum rate can be achieved by enabling D2D communications compared to the conventional cellular system. Necessary additions to an LTE-Advanced network to enable D2D session setup and management are proposed in [3].

On another front, recently, a new paradigm shift in the design of wireless systems has occurred where it has become evident that enhancing the proximity between the access network and the end users has the potential to provide the next performance leap in attainable rates via spatial spectrum reuse and to enhance indoor coverage as well [4]. LTE-Advanced provides means for deployment and planning of pico- and femto-cells, which are characterized by small transmission power and coverage radius thus enabling reuse of spectrum resources over a smaller area.

Another research trend that has potential to boost the overall cellular spectral efficiency is interference alignment (IA) [5]. IA offers the wireless interference channel with $K$ transmitterreceiver pairs the ability to simultaneously provide each user the opportunity to send at a data rate equal to half of his interferencefree channel capacity to his desired receiver, even though the number of users $K$ can be arbitrarily large. Cadambe and Jafar (CJ) [5] have shown that the achievable degrees of freedom are bounded by the number of symbol extensions, and it is possible to achieve $K / 2$ degrees of freedom per orthogonal time and frequency dimension as the number of channel extensions reaches infinity. This result allows the degrees of freedom to grow linearly with the number of users without cooperation in the form of message sharing thus allowing MIMO behavior.

The rest of this paper is organized as follows: Section II presents the system model and an overview of the proposed scheme. Section III presents the proposed clustering and IA grouping methods. Section IV presents the IA-based transmission scheme and the associated resource block allocation. Section V presents performance evaluation results and their discussion while Section VI concludes the paper and discusses future work.

\section{SYSTEM MODEL}

We consider an LTE-Advanced environment with a single sector hexagonal cell in isolation with radius $R$ with $N_{D 2 D}^{U s e r s}$ D2D users uniformly distributed over the cell and that all of these users are in active mode. There are $N_{D 2 D}^{\text {Pairs }}$ (equal to $N_{D 2 D}^{\text {Users }} / 2$ ) transmitters that need to communicate with $N_{D 2 D}^{\text {Pairs }}$ receivers, where $N_{D 2 D}^{\text {Pairs }}$ 


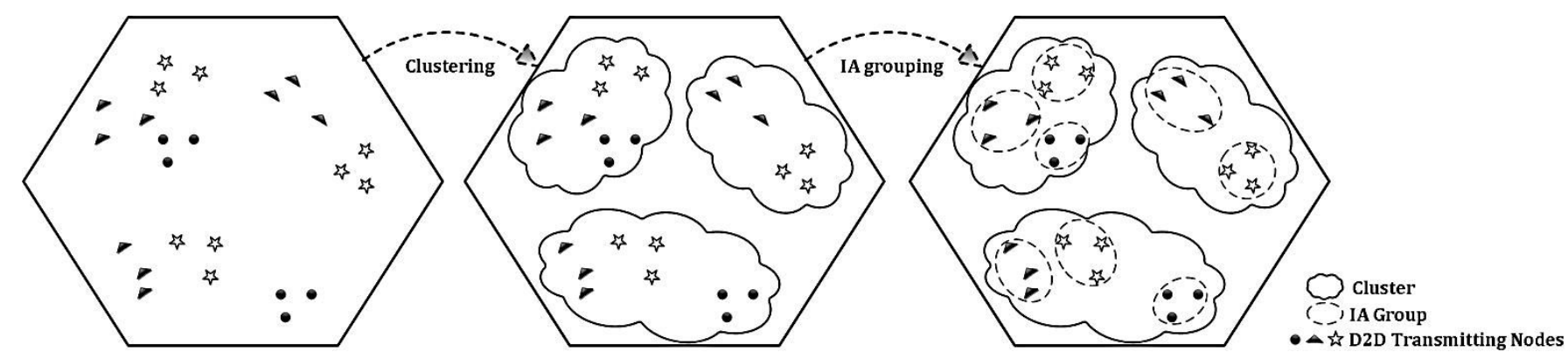

Figure 1. An illustrative example on the clustering and IA grouping steps

represents the number of $\mathrm{D} 2 \mathrm{D}$ pairs. We also assume that the maximum distance between the transmitter and receiver of each pair is constrained to $L_{\mathrm{Max}}$. Moreover, we assume a single omnidirectional antenna per user and we will only consider the D2D communication links. We assume that the D2D and macro-cell users are assigned orthogonal resources. Hence, no interference is experienced among them. We also assume the availability of $N^{R B}$ resource blocks (RBs), which are divided into $N_{\text {Macro }}^{R B} \mathrm{RBs}$ dedicated to macro-cell users, and $N_{D 2 D}^{R B} \mathrm{RBs}$ dedicated to D2D communication.

We propose a resource management scheme mainly based on shrinking the resource reuse distance by forming "clusters" of D2D pairs and fully reuse the available resource blocks over these clusters. In addition, within each cluster we assemble the D2D pairs into "IA groups" where each group is comprised of three D2D pairs. The reason for the choice of the group size to be three is to reduce the complexity of IA precoding. IA is then applied to the D2D links where resources are shared between pairs of the same IA group to further boost the cellular network spectral efficiency.

The example in Fig. 1 illustrates the main idea of the proposed scheme. Firstly, clusters of D2D pairs are formed. The formation of clusters is based on transmitting nodes positions and is done using what we refer to as the position-based scheme (PBS) that is based on the Fuzzy C-Means Clustering algorithm [6], which will be explained later in the sequel. Secondly, IA groups within each cluster are formed using channel gains and distances between transmitting and receiving nodes. Moreover, we assume that the base station is the entity responsible on running the clustering and grouping algorithms and then reporting them back to the D2D nodes. We propose two schemes for forming the IA groups:

1) The channel-based scheme (CBS) that is also based on the Fuzzy C-Means Clustering algorithm and which attempts to group $\mathrm{D} 2 \mathrm{D}$ pairs such that in each group there is a pair with high, intermediate, and low direct channel gain.

2) A simple distance-based scheme (DBS) that combines the benefits of ensuring both small containing areas for the IA groups and the existence of at least one pair of high channel gain in each of the IA groups.

Since both the clusters and IA group formations use the Fuzzy C-Means clustering mechanism, we provide some background and the motivation for using this concept in the following section.

Define $N^{C l}$ as the number of clusters of D2D pairs to be formed and $N_{u}^{C l}$ as the number of D2D pairs per cluster where $N_{u}^{C l}=\frac{N_{D 2 D}^{\text {Pairs }}}{N^{C l}}$. Furthermore, let $\mathbf{C}$ be an $N_{u}^{C l} \times N^{C l}$ matrix that contains the indices of the $N_{u}^{C l}$ pairs that belong to each of the
$N^{C l}$ clusters. Also, let the matrix $\mathbf{G}$ be a $3 \times N_{G}$ matrix containing the indices of the 3 pairs that form each IA group where $N_{G}=$ floor $\left(\frac{N_{D 2 D}^{\text {Pairs }}}{3}\right)$ is the number of IA groups. Note that if $N_{D 2 D}^{\text {Pairs }}$ is not an exact multiple of 3 , the remainder of the users will simply use point-to-point (P2P) transmission. We also define a group of matrices $\left\{\mathbf{D}_{n}, n=1,2, \cdots, N^{C l}\right\}$, each of which is an $N_{u}^{C l} \times N_{u}^{C l}$ matrix that contains the distances between different D2D users within cluster $n$, i.e., $d_{j k}^{n}$ represents the distance between transmitter $j$ and receiver $k, j, k \in\left[1, N_{u}^{C l}\right]$, located in cluster $n$.

\section{FUZZY CLUSTERING SCHEMES}

Cluster analysis divides data into groups (clusters) such that similar data objects belong to the same cluster and dissimilar data objects to different clusters. It has been used in Wireless Sensor Networks (WSN) by dividing the sensor networks into small manageable units to facilitate energy efficient routing and data reduction techniques. Moreover, it has advantages like conserving communication bandwidth within the clusters, avoiding redundant message transfer between the sensor nodes, and localizing energy efficient route setup within the clusters. However, in this paper, clustering is used to enable radio resources reuse over smaller areas and also to facilitate precoding for interference alignment at receivers when radio resources are shared to further enhance the overall system sum rate.

Fuzzy clustering provides a flexible and robust method for handling natural data with vagueness and uncertainty. In fuzzy clustering, each data point will have an associated degree of membership for each cluster. The membership value indicates the strength of its association to that cluster. The most prominent fuzzy clustering algorithm is the Fuzzy C-Means [6], which involves two processes: the calculation of cluster centroids as being the mean of all points, weighed by their degree of belonging to the cluster and the assignment of points to these centers using a form of Euclidian distance. This process is repeated until the cluster centers stabilize. The algorithm results in clusters of spherical shape and approximately the same size. In [6], performance comparison of Fuzzy K-Means, Fuzzy C-Means, Gaussian Mixture and Single-Link hierarchical clustering algorithms for different data sets is presented. The Fuzzy C-Means algorithm is shown to perform very well; in all datasets. The degree of correctness obtained in the categorization was comparable to the best ones achieved. Moreover, the performance speed was very acceptable. More specifically, for a data set with $N$ input patterns, the computational complexity of the Fuzzy CMeans clustering algorithm is near $O(N)$. The Fuzzy C-Means Clustering algorithm is shown in Fig. 2. In this algorithm, centroids represent the center of each cluster and each data point has a feature vector that represents the feature of the data point 
upon which we take the clustering decisions. This algorithm will be used for both cluster formations and IA group formations as explained in the following subsections.

\section{A. The D2D Clusters Formation}

To attain increasing data rates for the system, we aim at reducing the frequency reuse distance. Hence, to reuse the available $N_{D 2 D}^{R B}$ RBs, we cluster all the active D2D links depending on their positions in order to isolate them spatially and then explore the potential of boosting the spectral efficiency via decreasing the spatial-reuse distance in D2D environment. The positions of D2D nodes can be obtained by either a GPS or a positioning algorithm such as those in [7], [8]. Hence, we propose to use a Position Based Scheme (PBS) for the clustering process as it offers the ability to cluster D2D pairs in small containing areas. PBS is based on Fuzzy C-Means scheme by setting the feature vector to contain the positions of the D2D transmitting nodes. Here, the parameters $N_{c}$ and $N_{P}$ of Algorithm 1 are set to $N^{C l}$ and $N_{u}^{C l}$, respectively, where $N_{c}$ represents the final total number of centroids defined in the algorithm [6]. The $N_{u}^{C l} \times N^{C l}$ matrix $\mathbf{C}$ is updated column by column. The scheme is shown in Fig. 2, where the feature vector $\mathbf{F}$ values are the positions of the transmitting nodes whose indices are initially available in the set $\left\{1,2, \ldots, N_{D 2 D}^{\text {Pairs }}\right\}$, and is updated for each of the algorithm iterations. The modification of the C-Means clustering algorithm is to allow for an equal number of D2D pairs to be grouped in each of the clusters where in each of the iterations a number of $N_{P}$ pairs that have the highest degree of membership to the same centroid are chosen to form a cluster. This mechanism shows the ability to group D2D pairs in clusters characterized by nearly-separate small containing areas.

\section{B. The IA Group Formation in each Cluster}

In a normal P2P D2D-enabled cell operation, each transmitter will be assigned dedicated resources, e.g., a set of RBs to communicate with its corresponding receiver. However, to enable the usage of IA technique, we need to define users that will be grouped together and who will intentionally use the same resources in the manner defined by IA, which allows the extra degrees of freedom offered to be attained. In this subsection, we present two different grouping schemes as mentioned previously. In our model, we will assume that the size of each of the formed clusters $N_{u}^{C l}$ is a multiple of three. Hence, all D2D pairs shall be grouped.

We assume a set $\mathcal{P}$ that contains the indices of each D2D pair that has been assigned to an IA group, which is initialized as an empty set. We also define $N_{r}^{\text {Pairs }}$ as the number of remaining ungrouped D2D pairs, which is first initialized as $N_{u}^{C l}$. Without loss of generality, and to simplify the discussion of the grouping schemes, we assume that all D2D pairs belong to a single cluster, i.e., $N^{C l}$ is equal to one and $N_{u}^{C l}$ is equal to $N_{D 2 D}^{\text {Pairs }}$. Note that, in the case of multiple clusters, the steps are repeated per cluster with the proper definition of the parameters. We also note here that it is possible to define a position-based group formation scheme identical to that used in the clusters formation process. However, this did not provide good results and we omit it for the purpose of having a focused discussion. The two proposed grouping schemes are described below.

\section{1) Channel-Based Grouping Scheme (CBS)}

The first scheme, CBS, follows the same steps as those of Algorithm 1 shown in Fig. 2. The only difference from PBS is that the feature vector $\mathbf{F}$ now contains the gains of the direct channel between each D2D pair transmitter and receiver. The variable $N_{c}$ is initialized to three, and $N_{P}$ is set to $N_{G}$. The matrix of IA Groups $\mathbf{G}$ is updated row by row. Then, we calculate the average channel gain for all users existing in the same row of the matrix $\mathbf{G}$. What CBS does is that it categorizes all the available D2D pairs into three categories: D2D pairs with high, intermediate, and low direct channel gain. It then chooses a pair from each category to form an IA group. In the CBS scheme, we do not take the positions of users into consideration, i.e., users in a single group can be distributed over the whole cell/cluster area as long as the distance between the transmitter and receiver is less than $L_{\mathrm{Max}}$, as mentioned before. The main drawback of this grouping mechanism is that it might result in groups characterized by dispersed distribution of users over the cell/cluster where each group contains at least one pair with high channel gain. To be able to define a group over a small area, the following simple scheme is proposed.

\section{2) Distance-Based Grouping Scheme (DBS)}

The grouping criterion in DBS is based on attempting to select the D2D pairs in a group that are likely to cause large mutual interference on each other. When IA is applied, this mutual interference should be eliminated and thus, throughput gains can be achieved. The DBS assumes that the distance between the transmitter and receiver of each pair is a good measure of the channel path loss, which is the parameter that has the largest effect on the channel gain. Hence, we can say that DBS takes into account the channel conditions between the IA group lead pair's transmitter and receiver; and at the same time tries to contain each

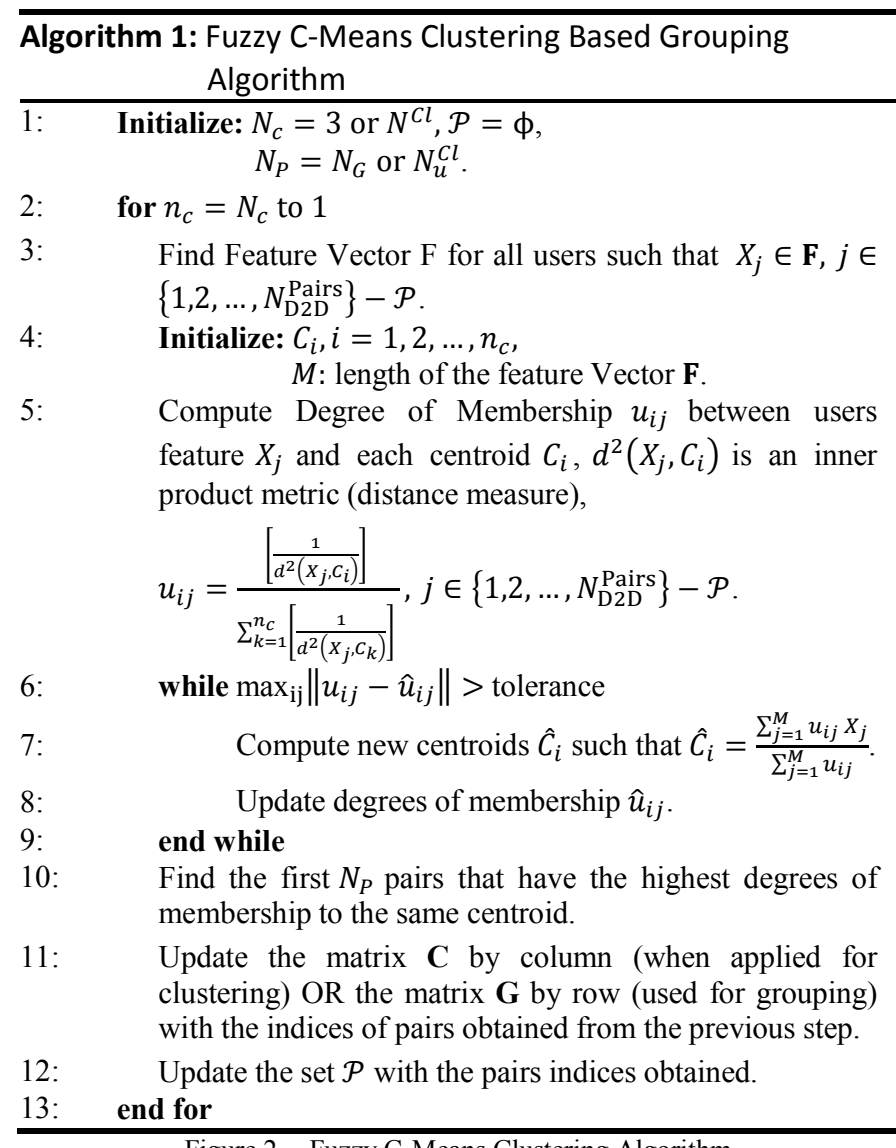

Figure 2. Fuzzy C-Means Clustering Algorithm 
IA group in a small area, where we define a lead pair as the pair that will be assigned the extra degrees of freedom offered by applying IA.

Algorithm 2 shown in Fig. 3 summarizes the procedure. We first choose a pair that has the minimum distance between its transmitter and receiver from the $\left\{\mathbf{D}_{n}, n=1,2, \cdots, N^{C l}\right\}$ group of matrices and set it as the lead pair in an IA group. Then, we choose two other pairs that have a minimum distance between their transmitters and the lead pair receiver.

\section{THE PROPOSED IA-BASED TRANSMISSION AND THE ASSOCIATED RESOURCE BLOCK ALLOCATION SCHEME}

Once the clusters are formed, all the resource blocks dedicated to D2D communication are fully reused in each cluster. The next problem to tackle would be how to apply IA in the formed IA groups within each cluster and which RBs will be allocated to members of the different IA groups. These two problems are discussed in the next two subsections.

\section{A. The Overall D2D IA-based Transmission Scheme}

In general, IA-based transmission schemes allow users to share their resources and align the interference caused by the sharing process through precoding. We group the D2D pairs, assign each group multiple RBs, and use IA to precode transmissions. Although the scheme is not limited to a group size of three pairs, we consider only the 3 -user interference channel with limited resources, $3 n_{r}$ RBs in each group, $n_{r} \in\{1,2,3, \ldots\}$. This allows $4 n_{r}$ simultaneous transmissions over the $3 n_{r}$ RBs. The limitation of 3-users per groups is imposed in order to cope with the practical limitations discussed in earlier precoding design works [5], which shows that the complexity of IA precoding calculations tremendously increases as the number of users and the required symbol extensions increase. The calculations of the precoding vectors are based on [5] and are discussed in [9] along with the design of the receivers decoders.

Afterwards, we choose the lead pair in each IA group to be the pair that has the highest average gain.

The system proposed so far depends on the centralized calculations of the precoding vectors. However, the results shown are encouraging to further evaluate the system performance when users are equipped with multiple antennas and distributed algorithms for precoding vectors calculations are considered such

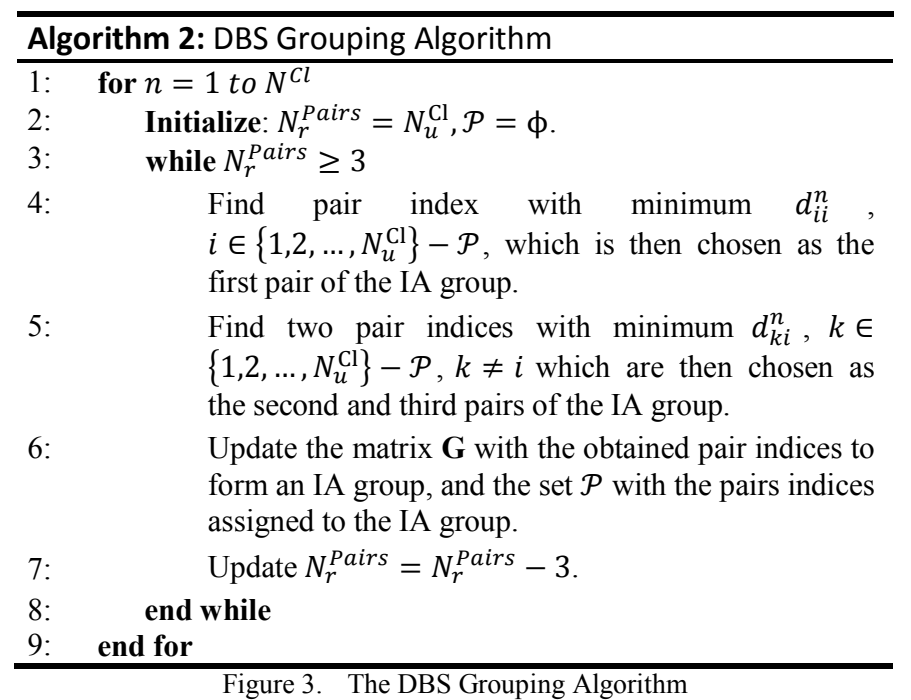

as that proposed in [10].

\section{B. Resource Block Allocation for the D2D Links}

We focus on the overall sum rate assuming no QoS or minimum rate requirements. Hence, we assume equal resource sharing between links, i.e., each link is assigned the same amount of RBs. In our model, each active link is assigned at least one RB. For a single cluster, each user will be assigned a number of RBs that is equal to $N_{D 2 D}^{R B} / N_{D 2 D}^{\text {Pairs }}$. For multiple clusters, the resource allocation process is performed for each cluster individually and all $N_{D 2 D}^{R B}$ RBs are allocated for each cluster allowing each user to be allocated a number of $\left(N_{D 2 D}^{R B} \times N^{C l}\right) / N_{D 2 D}^{\text {Pairs }}$ RBs.

A resource allocation optimization problem that aims at maximizing the sum of SINRs (which can be a good indication for realizable rates) for the D2D pairs in the formed clusters can be formulated as follows:

$$
\max \sum_{k=1}^{N_{D 2 D}^{R B}} \sum_{n=1}^{N^{C l}} \sum_{i=1}^{N_{u}^{C l}}\left(\frac{\alpha_{i, k}^{n} P\left|H_{i, i}^{k, n, n}\right|^{2}}{\sigma^{2}+\sum_{l=1, l \neq n}{ }^{C l} \sum_{j=1}^{N_{u}^{C l}} \alpha_{j, k}^{l} P\left|H_{i, j}^{k, n, l}\right|^{2}}\right)
$$

Subject to:

$$
\begin{aligned}
& \alpha_{i, k}^{n}, \alpha_{j, k}^{l}=\left\{\begin{array}{l}
1 \\
0
\end{array}\right. \\
& \sum_{i \in G_{s}} \alpha_{i, k}^{n}=\left\{\begin{array}{l}
3 \\
0
\end{array}, n=1,2, \ldots, N^{C l} ; k=1,2, \ldots, N_{D 2 D}^{R B}, s=\right. \\
& \sum_{k=1}^{N_{R B}} \alpha_{i, k}^{n} \geq 3, n=1, \ldots, N_{G},
\end{aligned}
$$

We assume that all D2D pairs are grouped in IA groups of three each. Moreover, we assume that $N_{D 2 D}^{R B} \geq N_{u}^{C l}$. In the above formulation, $\alpha_{i, k}^{n}$ is the selection variable that indicates the allocation of $\mathrm{RB} k$ for pair $i$ in cluster $n, H_{i, j}^{k, n, l}$ is the channel matrix between pair $j$ transmitter in cluster $l$ and pair $i$ receiver in cluster $n, G_{s}$ is the $s^{\text {th }}$ column of the matrix $\mathbf{G}$, and $\sigma^{2}$ is the noise variance. We also assume equal power sharing $P$ at all transmitters. The constraints ensure that all pairs in the same IA group are assigned the same RB and that each IA group shares at least three RBs. The aforementioned problem is very complex and methods for its efficient solution are left for future work. However, in a D2D setup it will be required to have simple and efficient methods due to the nature of the decision making process in such environment. We propose below reasonable allocation methods that can work in practice.

The first method distributes the $N_{D 2 D}^{R B}$ RBs over users in such a way that the users that have the best channel conditions are allocated resources first, which we call the "greedy best channel allocation". The second allocation method is based on proportional fair resource allocation in which we multiply the channel matrices by a metric and then allocate resources in the same way as the greedy allocation. The metric $m_{i k}$ is calculated as

$$
m_{i k}=\frac{C_{i k}}{R_{i}}
$$

where $R_{i}$ represents the average rate realized for the D2D pair $i$, and $C_{i k}$ represents the capacity of $\mathrm{RB} k$ for the pair $i$ and is calculated as

$$
C_{i k}=\omega_{i} B \log _{2}\left(1+\frac{\left|H_{i k}\right|^{2}}{\sigma_{i k}^{2}+I_{i k}}\right)
$$


where $\omega_{i}$ represents the degrees of freedom available for the pair $i$, which is equal to 1 for a normal pair and 2 for a lead pair, $B$ represents the bandwidth of a single $\mathrm{RB}, H_{i k}$ represents the channel coefficient for pair $i$ at $\mathrm{RB} k, \sigma_{i k}^{2}$ represents the noise variance for pair $i$ at $\mathrm{RB} k$, and $I_{i k}$ represents the last reported interference gain that pair $i$ suffered from at $\mathrm{RB} k$.

It is worth mentioning here that in $\mathrm{P} 2 \mathrm{P}$ transmission, each link uses the assigned RBs exclusively to transmit its message and in that case the grouping schemes are irrelevant.

\section{PERFORMANCE EVALUATION}

For the implemented simulation model, large indoor hall (WINNER B3 hotspot [11]) scenario is assumed for all direct and interfering links that have a maximum distance between the transmitting (interfering) node and the receiver of $L_{\mathrm{Max}}$. Furthermore, outdoor to indoor (WINNER B4) scenario is assumed for all interfering links with link distance greater than $L_{\text {Max }}$. The preceding assumptions are typical for large hall scenarios such as: airports, malls, libraries, and bookstores. Other simulation parameters are summarized in Table 1. Furthermore, the simulation results shown are the average of multiple simulation runs; each run is 1 second long. Users are distributed randomly over the cell. The users have very low mobility, which further complies with the assumed scenarios and the proposed applications such as providing internet services in public places. With these realistic assumptions, the clustering and grouping remain constant during each run. We believe that high mobility users should not be part of D2D and should rather be switched to normal cellular operation.

\section{A. Single cluster per cell}

For the D2D setup assuming a single cluster, comparison between the total cell D2D sum rate, when using P2P transmission and IA transmission, is shown in Fig. 4. The results reveal that the CJ-IA scheme provides opportunities for boosting D2D network spectral efficiency. The CBS is shown to have the best performance for the CJ-IA scheme over P2P transmission for all transmit signal power values. The DBS has a close performance to CBS performance. The maximum D2D sum rate achieved by the $\mathrm{P} 2 \mathrm{P}$ transmission is about $8.03 \mathrm{Mbps}$ while the IA transmission is able to achieve sum rate of about $10.59 \mathrm{Mbps}$; a gain of about $31.8 \%$.

In Fig. 5, we plot the Jain's fairness index for P2P transmission and CJ-IA schemes when using the different grouping algorithms. The figure shows that the gains in achievable rates provided by IA, typically associated with the high SNR regime, result in little penalty in the fairness performance. We note that the rates achieved by the lead pairs are normalized by the increase in offered degrees of freedom to have a meaningful comparison.

TABLE I. System LEVEl SimUlation PARAMETERS

\begin{tabular}{|c|c|}
\hline Parameter & Value \\
\hline Number of D2D pairs & 24 \\
\hline Cell radius $-R(\mathrm{~m})$ & 500 \\
\hline Noise power per RB $(\mathrm{dBm})$ & -121 \\
\hline Number of D2D RBs $-N_{D 2 D}^{R B}$ & 24 \\
\hline$L_{\mathrm{Max}}(\mathrm{m})$ & 80 \\
\hline Modulation Scheme & QPSK \\
\hline Number of Clusters $N^{C l}$ & $1,2,4$ \\
\hline Number of Users per Cluster $N_{u}^{C l}$ & $24,12,6$ \\
\hline
\end{tabular}

\section{B. Multiple clusters per cell}

In the case when multiple clusters are formed, a comparison between the total cell D2D sum rate for the different cluster sizes is shown in Fig. 6. The DBS grouping scheme is used for the IA grouping in all the formed clusters. The results in Fig. 6 show the large gains in overall rates that can be achieved by the reuse of resource blocks in the clusters. It is to be noted that if we normalize the total sum rates by the number of clusters $N^{C l}$, we can observe the effect of interference when resources are being reused among clusters as shown in Fig. 7. As the number of clusters increases, the effect of interference increases, but it is only effective for transmit signal powers greater than $-15 \mathrm{dBm} / \mathrm{user} / \mathrm{RB}$ for the scenario considered.

The results shown so far present the allocation based on greedy (Gr) best channel allocation for the RBs among the pairs. Figs. 8 and 9 show the comparison of sum rate and fairness index when using the greedy and the proportional fair resources allocation in the case where we assume 4 clusters per cell, each containing 6 D2D pairs, where (PF) denotes the proportional fair allocation results. We notice in Fig. 8 that there is a slight reduction in sum rate when using PF resource allocation. But, we can also see that fairness is improved for transmit powers less than $-15 \mathrm{dBm}$ in Fig. 9. The lack of improvement in fairness when using PF for transmit powers greater than $-15 \mathrm{dBm}$ is mostly due to the effect of interference between clusters that becomes more prominent when transmit power levels increase.

\section{CONCLUSIONS}

We have shown that the overall capacity of a D2D enabled cellular network using the separate resource sharing mode can be improved by using the IA technique and it suffices to take channel conditions between each pair's transmitter and receiver into account for grouping as in the CBS scheme to achieve the highest performance in a single cluster where interference is not an issue. Also, we have shown that for the proposed D2D scenarios, frequency reuse can achieve better sum rates that are proportional to the number of formed clusters. The interference induced by the reuse process is not very significant at signal transmit powers less than $-15 \mathrm{dBm}$ for the D2D setup provided. It can also be noted that IA can be implemented accurately without transmitter synchronization as detailed in [12]. Future work includes the relaxation of the assumption of the availability of full channel state information at the transmitters and receivers and implementing practical schemes for IA using the minimalist information expected to exist in mobile handsets. Also, the study of the effect of the parameter $L_{\mathrm{Max}}$ on the interference among clusters and the study of the relation between $L_{\mathrm{Max}}$ and the number of clusters within a cell and its effect on sum rate are worth investigating.

\section{REFERENCES}

[1] P. Janis, C.-H. Yu, K. Doppler, C. Ribeiro, C. Wijting, K. Hugl, O. Tirkkonen, and V. Koivunen, "Device-to-Device Communication Underlaying Cellular Communications Systems," Int'l J. of Communications, Network and System Sciences, vol. 02, no. 03, pp. 169-178, 2009

[2] C.-H. Yu, K. Doppler, C. Ribeiro, and O. Tirkkonen, "Performance impact of fading interference to Device-to-Device communication underlaying cellular networks," 2009 IEEE 20th International Symposium on Personal, Indoor and Mobile Radio Communications, pp. 858-862, Sep. 2009.

[3] K. Doppler, M. Rinne, C. Wijting, C. Ribeiro, and K. Hugl, "Device-todevice communication as an underlay to LTE-advanced networks," IEEE Communications Magazine, vol. 47, no. 12, pp. 42-49, Dec. 2009. 
[4] D. Lopez-Perez, I. Guvenc, G. De La Roche, M. Kountouris, T. Quek, and J. Zhang, "Enhanced intercell interference coordination challenges in heterogeneous networks," IEEE Wireless Communications, vol. 18 , no. June, pp. 22-30, 2011.

[5] V. R. Cadambe and S. A. Jafar, "Interference Alignment and Spatial Degrees of Freedom for the K User Interference Channel," 2008 IEEE International Conference on Communications, vol. 54, no. 8, pp. 971975, 2008.

[6] G. Fung, "A Comprehensive Overview of Basic Clustering Algorithms,' IEEE June, pp. 1-37, 2001.

[7] K. Y. K. Yu and E. Dutkiewicz, Geometry and Motion Based Positioning Algorithms for Mobile Tracking in NLOS Environments, vol. 11, no. 2. IEEE, 2010, pp. 1-5.

[8] N. Sirola, Closed-form algorithms in mobile positioning: Myths and misconceptions, no. x. Ieee, 2010, pp. 38-44.

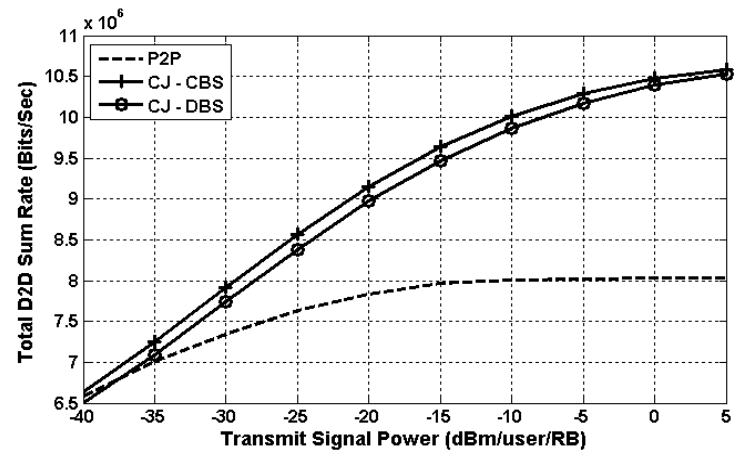

Figure 4. Total sum rate of a single cell enabling D2D communication for both P2P and IA transmission.

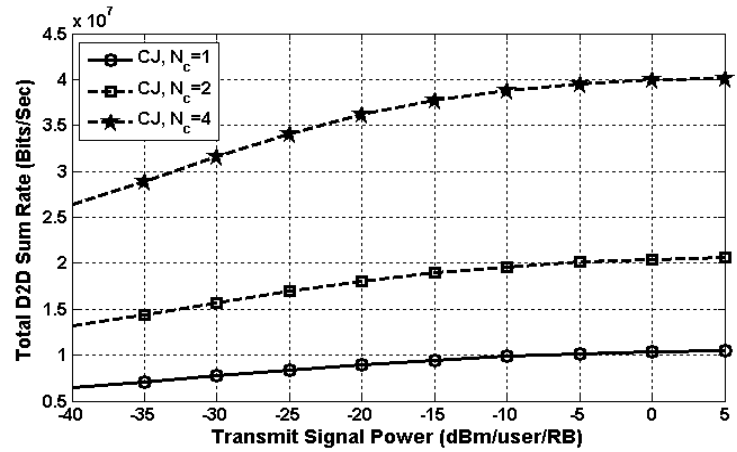

Figure 6. Total sum rate of a single cell enabling D2D communication with IA transmission for different cluster sizes.

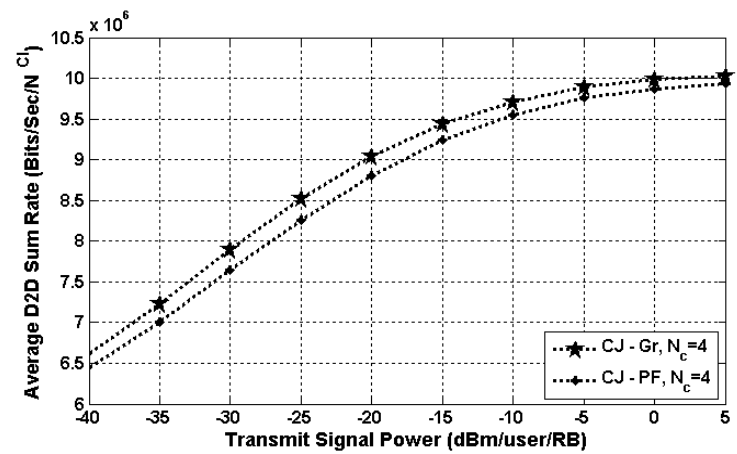

Figure 8. Comparing total sum rate per cluster for greedy and proportional fair resources allocation.
[9] H. E. Elkotby, K. M. F. Elsayed, and M. H. Ismail, "Exploiting interference alignment for sum rate enhancement in D2D-enabled cellular networks," in 2012 IEEE Wireless Communications and Networking Conference (WCNC), 2012, pp. 1624-1629.

[10] K. Gomadam, V. R. Cadambe, and S. A. Jafar, "Approaching the Capacity of Wireless Networks through Distributed Interference Alignment," in IEEE GLOBECOM 2008 - 2008 IEEE Global Telecommunications Conference, 2008, pp. 1-6.

[11] D. Laselva, "Matlab SW documentation of WIM2 model," vol. 1, no. 20 pp. 1-20, 2008

[12] S. Gollakota, S. D. Perli, and D. Katabi, "Interference alignment and cancellation," ACM SIGCOMM Computer Communication Review, vol. 39 , no. 4, p. 159, 2009.

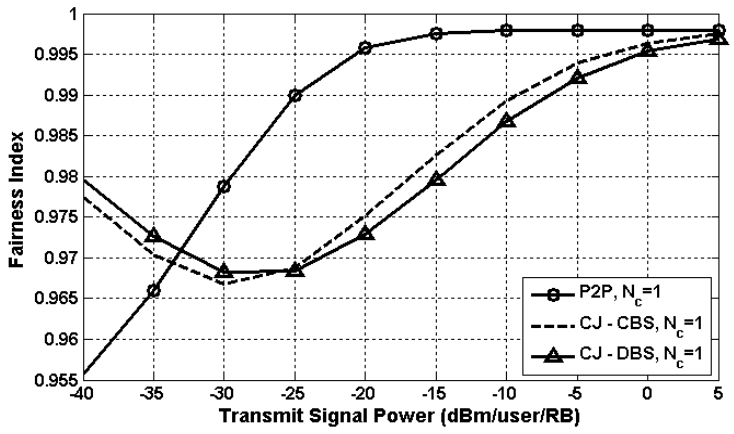

Figure 5. Fairness index results for both $\mathrm{P} 2 \mathrm{P}$ and IA transmission when using a) CBS. b) DBS. c) PBS

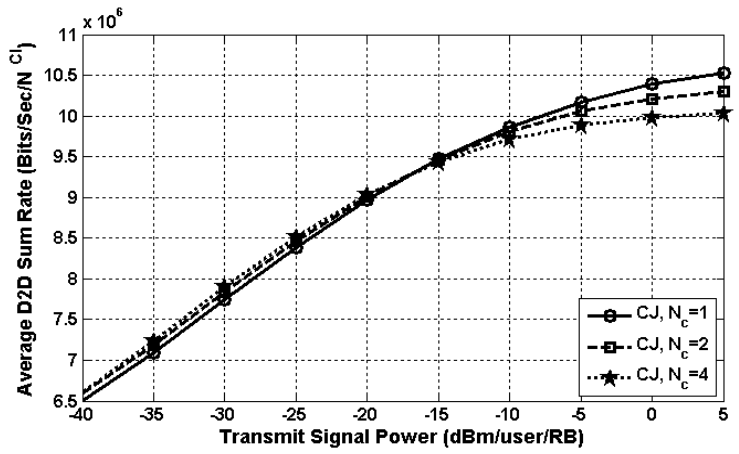

Figure 7. Total sum rate of a single cell enabling D2D communication with IA transmission for different cluster sizes normalized by the number of clusters $\mathrm{N}^{\mathrm{Cl}}$.

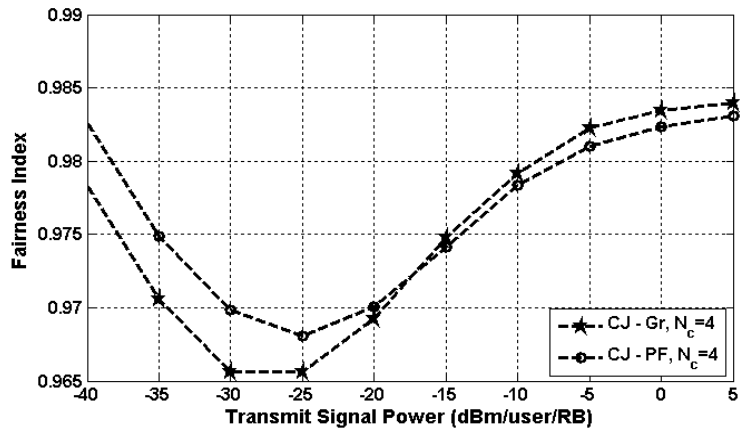

Figure 9. Comparing fairness index for greedy and proportional fair resources allocation. 